\title{
Patterns of relapse following hippocampal avoidance prophylactic cranial irradiation for small cell lung carcinoma
}

\author{
Theresa A. Cook ${ }^{1}$, Matthew R. Hoffmann ${ }^{2}$, Amie J. Ross ${ }^{1}$, Kirsty J. Turnbull ${ }^{1}$, Justin Westhuyzen ${ }^{1}$, \\ Noel J. Aherne $e^{1,3,4}$ \\ ${ }^{1}$ Department of Radiation Oncology, Mid-North Coast Cancer Institute, Coffs Harbour, New South Wales, Australia \\ ${ }^{2}$ Department of Radiation Oncology, Mid-North Coast Cancer Institute, Port Macquarie, New South Wales, Australia \\ ${ }^{3}$ Rural Clinical School, Faculty of Medicine, University of New South Wales, Coffs Harbour, New South Wales, Australia \\ ${ }^{4}$ School of Health and Human Sciences, Southern Cross University, Coffs Harbour, New South Wales, Australia
}

\begin{abstract}
Background: Hippocampal avoidance techniques are an evolving standard of care for patients undergoing cranial irradiation. Our aim was to assess the oncological outcomes and patterns of failure following hippocampal avoidance prophylactic cranial irradiation (HA-PCl) as a standard of care in unselected patients with both limited and extensive stage small cell lung carcinoma.

Materials and methods: Consecutive patients with small cell lung carcinoma with a complete (limited stage) or good partial (extensive stage) response following chemotherapy were eligible to receive $\mathrm{HA}-\mathrm{PCl}$, with a total dose of 25 Gray in $10 \mathrm{frac}-$ tions. All patients had a negative baseline MRI brain scan with gadolinium prior to $\mathrm{HA}-\mathrm{PCl}$. Patients had baseline and follow up Common Toxicity Criteria Adverse Event assessments. Following completion of HA-PCl, all patients had three-monthly MRI brain scans with gadolinium until confirmation of intracranial relapse, as well as three-monthly CT of the chest, abdomen and pelvis. Overall and progression-free survival were calculated using the Kaplan-Meier method.

Results: A total of 17 consecutive patients, 9 men and 8 women, with a mean age of 70 years received $\mathrm{HA}-\mathrm{PCl}$ between May 2016 and June 2020 after completion of their initial chemotherapy. There were no Grade 4 or greater adverse events. No patient had an isolated hippocampal avoidance zone relapse alone; three of 17 patients had multifocal relapses that included the hippocampal avoidance zone.

Conclusion: In our series, there were no hippocampal only relapses and we conclude that $\mathrm{HA}-\mathrm{PCl}$ is a safe alternative to standard PCl in the setting of small cell lung cancer.
\end{abstract}

Key words: hippocampus; hippocampal avoidance; prophylactic cranial irradiation; small cell lung cancer Rep Pract Oncol Radiother 2021;26(6):968-975

\section{Introduction}

In Australia, approximately 13,300 people were diagnosed with lung cancer in 2020, of which small cell lung carcinoma (SCLC) accounted for approximately $15 \%$ of all cases diagnosed annually [1]. The incidence of SCLC is increasing and overall prognosis remains poor. One third of cases have limited disease confined to the chest and are managed with concurrent chemo-radiation, with prophylactic cranial irradiation (PCI) reserved for those who have a complete clinical response. Two thirds of

Address for correspondence: Assoc. Prof. Noel Aherne, Department of Radiation Oncology, Mid-North Coast Cancer Institute, 345 Pacific Highway, Coffs Harbour, NSW 2450, Australia, tel: (02) 6656 5776, fax: (02) 6656 5797; e-mail: noel.aherne@health.nsw.gov.au

This article is available in open access under Creative Common Attribution-Non-Commercial-No Derivatives 4.0 International (CC BY-NC-ND 4.0) license, allowing to download articles and share them with others as long as they credit the authors and the publisher, but without permission to change them in any way or use them commercially 
cases have extensive stage disease and are managed initially with chemotherapy, with consolidative thoracic irradiation and PCI reserved for those with at least a good partial response. Given the high preponderance of brain metastases, PCI has been a cornerstone of treatment for over twenty years [2]. Given the potential for treatment related neurotoxicity, alternative treatment modalities including hippocampal avoidance techniques, as well as close MRI surveillance with early salvage with stereotactic radiation are being investigated in prospective clinical trials.

Small cell lung carcinoma has an aggressive biological behaviour and early brain metastases are common, with up to $50 \%$ of patients developing intracranial metastases during follow up [3]. Median survival for patients with limited stage disease is $18-24$ months, while for those with extensive stage disease median survival is approximately 12 months.

Recent improvements in therapy, including earlier detection through lung cancer screening programs, improved technological advancements in the delivery of radiation therapy, improved symptom management as well as the increasing use of immunotherapy, will likely result in a longer median survival for future patients. Thus, the multidisciplinary management of intracranial metastases and the need to balance their management with the risk of late toxicities - which patients may live to develop - has become progressively more important with time.

Historically, PCI has been demonstrated to reduce the incidence of brain metastases by up to $50 \%$, but at the cost of impairment of neurocognitive function as well as a reduction in health-related quality of life (QoL). This radiation induced neurocognitive decline is thought to be due to depletion of the neural stem cell population in the subgranular zone of the hippocampal dentate gyrus. The hippocampi are paired structures, one on each side of the brain which are part of the limbic system, and they play a critical role in the consolidation of short-term memory to long-term memory. The hippocampi also facilitate spatial memory to allow navigation and may be damaged during cranial radiation. With increasing knowledge of the role of hippocampus in the consolidation of short term memory [4], attention has turned to hippocampal avoidance PCI, based on the initial work of Gondi et al. [5]. While randomised trials on the utility of HA-PCI versus PCI are still in progress, many centres offer HA-PCI as a standard of care and it has been in use at our institution for patients undergoing cranial irradiation since 2016.

Earlier trials and meta-analyses in patients with limited stage disease supported the use of PCI through a clear demonstration of a reduction in the development of brain metastases as well as the impression of a specific overall survival advantage [2]. Similar data was published regarding patients with extensive stage disease when the EORTC published their trial in 2007 [6]. The trial established the use of PCI by demonstrating a two-thirds reduction in the incidence of brain metastases, as well as an almost doubling of the 1 year overall survival in these patients, with around $13 \%$ in the control arm versus $27 \%$ in the PCI arm, hazard ratio $(\mathrm{HR})=0.68$, $\mathrm{p}=0.003[6]$.

Conflicting results regarding a possible survival advantage came to the fore with the publication of the Japanese Clinical Oncology Group study [7]. Takahashi et al. randomised patients with extensive-stage disease to either upfront PCI or to magnetic resonance imaging (MRI) brain surveillance following completion of first line chemotherapy. The study found no difference in overall survival between the two arms, and the study was halted early as a consequence. The conflicting results between these two trials may be explained in a large part by the utilisation of rigorous MRI screening at trial entry, as well as differences within the trial populations. Based on these findings, there has been a new focus on moving away from conventional PCI. Alternatives including close MRI surveillance and the omission of PCI with use of radiosurgery on relapse [8], the addition of memantine as a neuroprotective agent [9], and use of novel agents such as atezolizumab are all under investigation or entering clinical use [10].

In the present study, we report on the oncological outcomes and patterns of failure following HA-PCI as a standard of care in unselected SCLC patients treated at a regional cancer centre.

\section{Materials and methods}

From May 2016 to June 2020, patients at our regional cancer institution with SCLC without progression after initial therapy were offered PCI. 


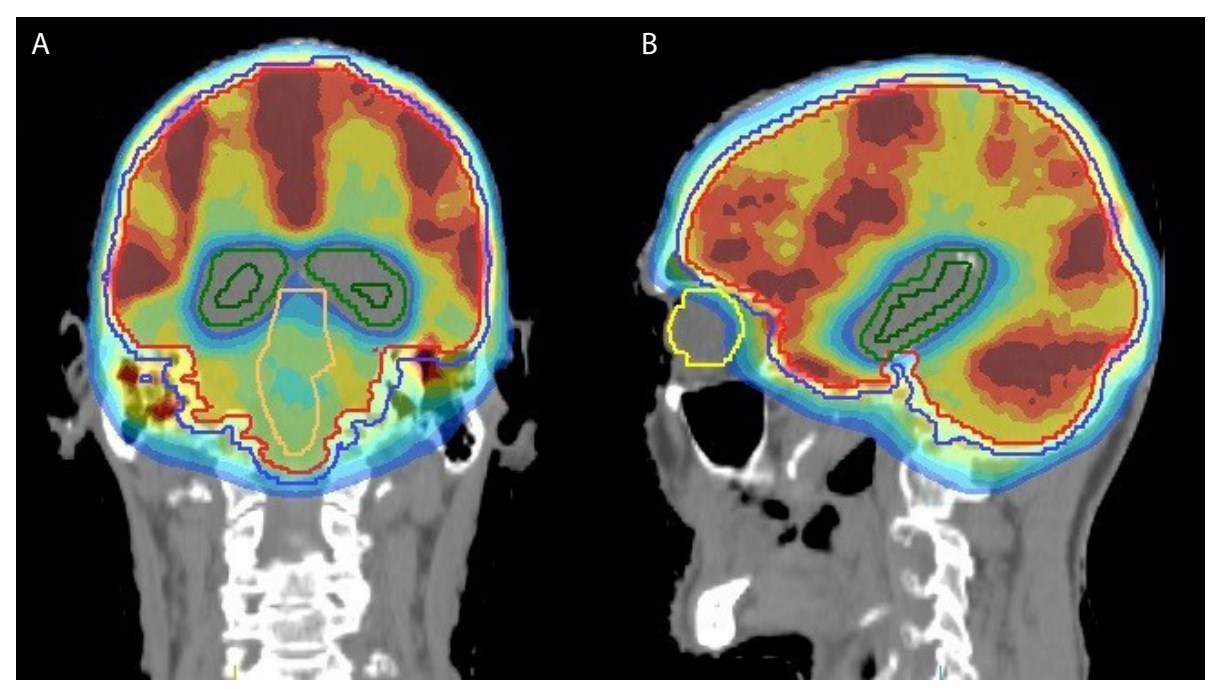

Figure 1. Sample isodose distribution above the $50 \%$ isodose on a hippocampal avoidance prophylactic cranial irradiation ( $\mathrm{HA}-\mathrm{PCl}$ ) plan in (A) coronal and (B) axial views. Outlined structures are hippocampi (dark green), hippocampal avoidance zones (mid green), brain-stem (orange), orbit (yellow), clinical target volume (CTV) (red), planning target volume (PTV) (blue)

Following informed consent discussion regarding the evolving evidence of potential cognitive benefits versus relapse risk of conventional PCI versus hippocampal avoidance PCI (HA-PCI), all patients were offered the choice of either treatment modality. Local department protocol required patients undergoing HA-PCI to have a MRI brain scan $(1.25 \mathrm{~mm}$ slices with $3 \mathrm{D}$ reconstruction and intravenous gadolinium contrast) to exclude the presence of brain metastases pre-treatment as per the RTOG 0933 radiation therapy planning guidelines [11]. This protocol also allowed accurate delineation of the hippocampi during radiation therapy planning, and for patients to undergo post-treatment 3-monthly MRI brain scans to allow for early salvage where intracranial relapse has been demonstrated.

\section{Radiation therapy procedure}

All patients undergoing HA-PCI were treated using volumetric modulated arc therapy (VMAT) technique with 6 Megavoltage photons to a total dose of 25 Gray in 10 fractions, once daily over 2 weeks, prescribed as per ICRU 83 [12]. Simulation included the use of reinforced thermoplastic mask, head rest and knee rest to ensure accurate reproducibility of set up. The hippocampi were volumed using the fused pre-treatment MRI, and a standard $5 \mathrm{~mm}$ isotropic expansion was used to create the hippocampal avoidance zone (HAZ) as per RTOG 0933 [5]. The clinical target volume
(CTV) was created by voluming the whole brain, and a $3 \mathrm{~mm}$ isotropic expansion was used to create the whole brain planning target volume (PTV). A bi-directional VMAT arc in the transverse plane and a non-coplanar bi-directional VMAT arc in the sagittal plane were used to deliver dose to the PTV while sparing dose to the hippocampi. An HA-PCI plan in coronal and axial views is illustrated in Figure 1.

Planning aims were based on those used in the study by Redmond et al. [13]: mean hippocampal dose $<8 \mathrm{~Gy}$, and minimum $90 \%$ of prescribed dose to be delivered to $90 \%$ of the whole brain target. Additional constraints included hippocampal maximum dose < 12 Gy, PTV D2\% < 120\% TD (minor violation $<125 \% \mathrm{TD}$ ), PTV D80\% > TD, lenses max dose $<5$ Gy (minor violation $<10 \mathrm{~Gy}$ ), orbits max dose $<25 \mathrm{~Gy}$ (minor violation $<30 \mathrm{~Gy}$ ), and brainstem, optic chiasm and optic nerves max dose $<\mathrm{TD}$ (minor violation $<125 \% \mathrm{TD}$ ). All patients had daily image guidance with cone-beam CT.

The electronic medical record (Mosaiq ${ }^{\circledR}$, Elekta, Crawley, United Kingdom) of all patients was retrospectively reviewed for dates of diagnosis, treatment dates, date and site of first progression, first intracranial progression, first hippocampal progression, death, and date of last follow up. We also reviewed all recorded toxicity assessments for all patients as well as parsing of the electronic medical record for qualitative evidence of any gross neurocognitive deficit. For those patients with intracranial relapse, 


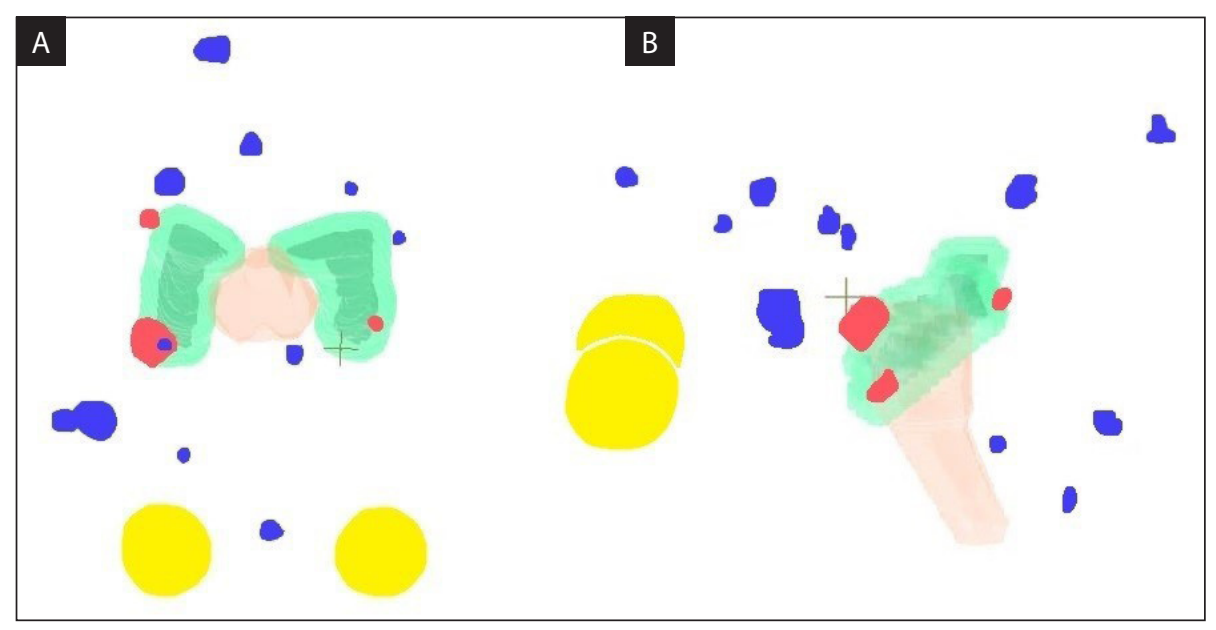

Figure 2. Composite image demonstrating location of brain metastases in the (A) axial and (B) sagittal planes. The orbits (yellow), brainstem (orange), hippocampi (dark green), and hippocampal avoidance zones (mid green) are outlined for orientation. Metastases overlapping hippocampal avoidance zones are red, metastases not overlapping hippocampal avoidance zones are blue

the MRI from first intracranial relapse was fused to the patient's original plan to determine the location of first intracranial progression, and whether it was within or outside the hippocampal avoidance zone. A composite plan of the surrogate patient with the median whole brain CTV volume in our series was used to demonstrate the hippocampal or non-hippocampal location of all brain metastases for all patients in our series (Fig. 2).

\section{Neurocognitive assessment}

Baseline and interval assessments of the cognitive disturbance and concentration impairment adverse event domains of the Common Toxicity Criteria Adverse Events (CTCAE) v4.0 [19] were available for all patients. Qualitative information was parsed from the electronic medical record to supplement this. As this was purely a retrospective analysis and not a clinical trial, no additional neurocognitive or QoL instruments were utilised over and above our normal standard of care.

\section{Statistical procedures}

MedCalc v19 0.4 (Ostend, Belgium) was used to perform Kaplan-Meier analysis of time to first intracranial progression, progression-free and overall survival. Progression-free survival (PFS) and overall survival (OS) were calculated from the commencement of treatment to progression or last follow up, or to death or last follow up, respectively.

\section{Ethical approval}

This retrospective study received approval from the North Coast New South Wales Human Research Ethics Committee (reference 2019/ETH12669).

\section{Results}

Between March 2016 and June 2020, 17 patients with SCLC met the criteria for inclusion in this retrospective study, and all had at least one post-treatment MRI brain scan available for review. Patient demographics for the cohort are displayed in Table 1. One patient was $>80$ years; this patient was treated on the basis of their clinical factors and an ECOG status of 1 . All patients with limited stage SCLC had platinum-etoposide chemotherapy concurrent with thoracic radiotherapy (50 Gray / 25 fractions) prior to PCI. Patients with extensive stage SCLC had initial platinum-etoposide chemotherapy, with subsequent decision on sequencing of thoracic consolidation and PCI individualised to the patient (Tab. 1). No patient had immunotherapy as part of their initial therapy.

Target brain coverage (brain D90 > 90\%) was met in all patients. Regarding organ at risk constraints, no patient had a major planning violation. Seven patients had minor violations - within $0.5 \mathrm{~Gy}$ on PTV D80 - and one patient had a minor violation on PTV D2 (PTV D2 = 121\%). Lenses met ideal constraints $50 \%$ of the time, and orbits met constraints in all plans. Minor violations for the 
Table 1. Patient characteristics

\begin{tabular}{|l|c|}
\hline Characteristic & $\begin{array}{c}\text { Number (\%)/ } \\
\text { /median (range) }\end{array}$ \\
\hline $\begin{array}{l}\text { Extense } \\
\text { Limited Stage }\end{array}$ & $10(59 \%)$ \\
\hline Age at diagnosis (years) & $7(41 \%)$ \\
\hline Gender & $70.0(58.5-83.6)$ \\
Male & \\
Female & $9(53 \%)$ \\
\hline ECOG prior to HA-PCI & $8(47 \%)$ \\
0 & \\
1 & $7(41 \%)$ \\
2 & $7(41 \%)$ \\
\hline Sequencing of thoracic consolidation & $3(18 \%)$ \\
and PCI for extensive stage & \\
No thoracic consolidation & $4(40 \%)$ \\
Sequential (PCI first) & $3(30 \%)$ \\
Sequential (thoracic first) & $2(20 \%)$ \\
Concurrent & $1(10 \%)$ \\
\hline
\end{tabular}

ECOG — Eastern Cooperative Oncology Group; HA-PCI — hippocampal avoidance prophylactic cranial irradiation

brainstem, optic chiasm and optic nerves were seen in some patients, occurring in two-thirds of plans. Mean hippocampal dose constraints were met in 13 of 17 patients as prioritisation was given to adequate PTV coverage and avoidance of excessive D2 dose. Hippocampal maximum dose constraints were met in 15 of 17 patients - hippocampal dose constraint violations were more commonly seen in our first patients treated, including a patient with the minor violation on PTV D2.

After a median follow up of 11.6 months (range 3.3-43.1), 8 patients showed intracranial progression $(47 \%)$ of whom 3 had their initial relapse in the hippocampal avoidance zone (HAZ) (38\% of intracranial recurrences, $18 \%$ of all patients). No patient with an initial HAZ relapse had an isolated HAZ relapse alone. A fourth patient with initial non-hippocampal only recurrence had later intracranial progression with both hippocampal- and non-hippocampal disease. Regarding extracranial progression, four patients (24\%) showed progression after PCI. Pictorial representations of typical isodose distribution and locations of metastases on first intracranial relapse are demonstrated in Figures 1 and 2 .

For the $8(47 \%)$ patients with intracranial relapse, the median time from HA-PCI to intracranial progression was 4.2 months (range 0.3 to 16.2 months). The Kaplan-Meier survival curves are shown in Figure 3. Four of these patients underwent salvage therapy at first and subsequent relapses with up to 4 episodes of intracranial salvage per patient. Median time from HA-PCI to intracranial progression or last follow up was 5.9 months (range 0.3 to 43.1 months). Progression of disease in any location was seen in 15 patients (88\%), with
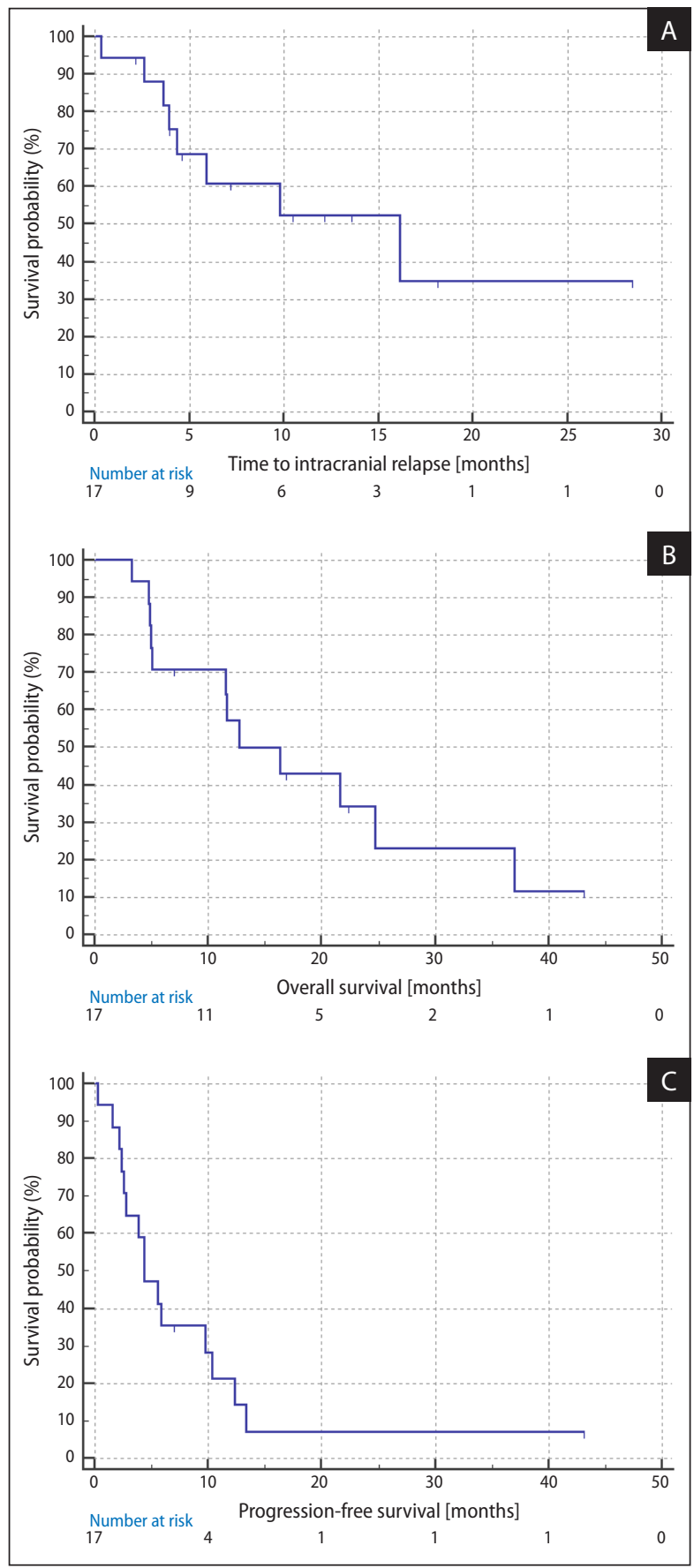

Figure 3. Kaplan-Meier survival probabilities. A. Time to intracranial relapse; B. Progression-free survival; C. Overall survival 
a median time to progression or last follow up of 4.3 months (range 0.3 to 43.1 months). Twelve patients had died by the time of retrospective review; median time from completion of HA-PCI to death in these patients was 11.6 months (range 3.3 to 37.0 months). Median survival for all patients - time to death or last follow up - was 11.6 months (range 3.3 to 43.1 months).

Fourteen of 17 patients (82\%) had a recorded assessment of cognitive disturbance as per CTCAE available, with an average of two assessments per patient. Of these, the highest recorded score at any time-point was 1 (mild cognitive disability, no specialised services required) for 4 patients, and 0 (no cognitive disturbance) for 10 patients. To supplement the CTCAE cognitive and concentration domains, a review of the oncology electronic medical record was undertaken to identify further qualitative information:

- one patient was noted to have mild word-finding difficulty during their HA-PCI course which resolved post-treatment and was likely related to lethargy, with no subsequent cognitive disturbances seen;

- one patient with intracranial and HAZ relapse and 4 subsequent courses of salvage therapy had confusion noted in their chart within 1 month of death secondary to intracranial progression;

- one patient without intracranial progression was noted to develop multifactorial delirium (secondary to hypercalcaemia, constipation and urinary retention) on 2 occasions within 4 months of death, starting from 6 months after their first extracranial progression;

- one patient with hippocampal and HAZ relapse was noted to develop increasing difficulties with word-finding and forgetfulness 17 months following HA-PCI;

- one patient without any intracranial relapse complained of increased forgetfulness at the 3-month follow up, with no further cognitive disturbance noted at subsequent follow up;

- one patient without any intracranial relapse was noted to have increased forgetfulness on most recent follow up 8 months after completion of HA-PCI.

The remaining 11 patients had no recorded issues with cognitive disturbance at any time during their treatment or follow-up.

\section{Discussion}

We report the first Australian results of patterns of failure following HA-PCI following initial therapy for SCLC in a regional cancer centre. Of 17 patients who received HA-PCI in our single institution, 8 (47.1\%) experienced intracranial progression after a median follow up of 11.5 months, with 3 cases of initial relapse including the HAZ, all of whom had synchronous metastases in the fully-dosed brain. Despite a heterogeneous patient group, and the majority of patients presenting with extensive stage SCLC ( 10 of $17 ; 59 \%$ ), a 13.7 month median survival may indicate that patients undergoing HA-PCI will live long enough to see the benefits of HA-PCI should the putative benefits be borne out in a randomised clinical trial.

Our current study adds to the emerging body of evidence on HA-PCI from RT0G 0933 [5] and, more recently, from Redmond et al. [13], OC-0503 [14], and SAKK 15/12 (15). RTOG 0933 [5] randomised 110 patients who already had brain metastases at least $5 \mathrm{~mm}$ beyond the hippocampi to whole brain radiotherapy or hippocampal-avoidance whole brain radiotherapy. As these patients were already known to have metastatic disease they represent a high risk for disease progression, and 67 of 110 patients developed intracranial progression but, of note, only 3 progressed within the HAZ; however, it was not reported whether this represented isolated recurrence or was synchronous with failure elsewhere in the brain.

In the 2017 cohort of 20 patients receiving HA-PCI for limited stage SCLC by Redmond et al., after a median follow up of 93 months, just 4 patients had developed brain metastases [13]. Half of these had first progression in both the fully-dosed and low-dosed regions of the brain, with no isolated hippocampal metastases similar to our series. In the OC-0503 trial [14], 168 patients with either limited or extensive stage SCLC were randomised to HA-PCI or standard PCI; of 19 patients with intracranial relapse after a median follow up of 24.6 months, none had isolated recurrences within the HAZ. In SAKK 15/12, 3 of 42 patients with limited stage SCLC had developed intracranial relapse within 12 months with no recurrences in the HAZ. The utility of HA-PCI in regards to intracranial control as an intervention in larger randomised trials is currently in progress. This is expected to 
Table 2. Published isodose constraints for hippocampal-avoidance PCI

\begin{tabular}{|c|c|c|}
\hline Study & Hippocampal constraints & Brain constraints \\
\hline \multicolumn{3}{|l|}{ Published trials } \\
\hline RTOG 0933 (2014) [5] & $\begin{array}{l}\mathrm{D} 100 \% \leq 9 \mathrm{~Gy} \\
\mathrm{Dmax} \leq 16 \mathrm{~Gy}\end{array}$ & $\begin{array}{c}\mathrm{CTV}=\text { whole brain }-\mathrm{HAZ} \\
\text { PTV D2\% } \leq 37.5 \mathrm{~Gy} \\
\text { PTV D95\% } \geq 25 \mathrm{~Gy}\end{array}$ \\
\hline Redmond (2017) [13] & Mean < 8 Gy & Whole brain D $90 \%>90 \%$ TD \\
\hline OC-0503 (2019) [14] & $\begin{array}{c}\text { Mean }<8.5 \mathrm{~Gy} \\
\mathrm{D} 1 \%<10 \mathrm{~Gy}\end{array}$ & $\begin{array}{c}\text { Dmax }<28.75 \text { Gy }(115 \%) \\
\text { V115\% < 1\% PTV }\end{array}$ \\
\hline SAKK 15/12 (2020) [15] & $\begin{array}{l}\mathrm{D} 98 \%<10 \mathrm{~Gy} \\
\mathrm{Dmax}<10 \mathrm{~Gy}\end{array}$ & Whole brain D95\% > 95\% TD \\
\hline \multicolumn{3}{|l|}{ Trials in recruitment } \\
\hline PREMER Trial [16] & $\begin{array}{l}\mathrm{D} 100 \% \leq 9 \mathrm{~Gy} \\
\mathrm{Dmax} \leq 16 \mathrm{~Gy}\end{array}$ & $\begin{array}{l}\mathrm{D} 2 \%<26.7 \mathrm{~Gy} \\
\mathrm{D} 98 \%>23.7 \mathrm{~Gy}\end{array}$ \\
\hline NCT02906384 [17] & $\begin{array}{c}\text { HAZ: } \\
\text { Dmin }<9 \text { Gy } \\
\text { Dmax }<16 \text { Gy }\end{array}$ & \\
\hline NRG-CC003 [18] & $\begin{array}{l}\mathrm{D} 100 \% \leq 7.5 \mathrm{~Gy} \\
\mathrm{Dmax} \leq 13.5 \mathrm{~Gy}\end{array}$ & $\begin{array}{c}\mathrm{CTV}=\text { whole brain }-\mathrm{HAZ} \\
\text { PTV V25 Gy }>95 \% \\
\text { PTV D2\% } \leq 31.25 \mathrm{~Gy} \\
\text { PTV D } 98 \% \geq 21 \mathrm{~Gy}\end{array}$ \\
\hline
\end{tabular}

In the SAKK 15/12 trial the expansion from the hippocampi to the hippocampal avoidance zones was $2 \mathrm{~mm}$, where specified all other trials used a $5 \mathrm{~mm}$ expansion [15]. CTC — clinical target volume; HAZ — hippocampal avoidance zone; PTV — planning target volume

more accurately quantify the potential neurocognitive benefit of this technique. As with any new technique, there is a learning curve for the entire multidisciplinary team to achieve specified dose criteria for the hippocampal avoidance region as based on Redmond et al. [13]. For comparison, we present the current published constraints in tabulated form (Tab. 2). Trials in recruitment are also listed [16-18].

Limitations of our study are the small numbers, given single centre only experience, and lack of more extensive neuropsychological cognitive assessment. We have attempted to mitigate this through recording of CTCAE cognitive domain adverse events as well as qualitative assessment. As there were no hippocampal only relapses in our series, we consider HA-PCI to be a safe alternative to standard PCI in the setting of SCLC. Future directions will include the potential for either HA-PCI as the new standard of care, or close MRI surveillance with focussed stereotactic radiation therapy as salvage.

This work was presented in part at the American Society of Clinical Oncology / American Society of Therapeutic Radiation Oncology Multidisciplinary
Thoracic Cancer Symposium, San Francisco, March 2017.

\section{Acknowledgments}

We would like to thank Dr Rahim Tahir for his early input into the development of the HA-WBRT protocol in our department.

\section{Conflict of interest}

None declared

\section{Funding}

None declared.

\section{References}

1. Australian Institute of Health and Welfare 2020. Lung cancer in Australia statistics 2020. https://www.canceraustralia.gov.au/affected-cancer/cancer-types/lung-cancer/ statistics.

2. Aupérin A, Arriagada R, Pignon JP, et al. Prophylactic cranial irradiation for patients with small-cell lung cancer in complete remission. Prophylactic Cranial Irradiation Overview Collaborative Group. N Engl J Med. 1999; 341 (7): 476-484, doi: 10.1056/NEJM199908123410703, indexed in Pubmed: 10441603.

3. Wolfson AH, Bae K, Komaki R, et al. Primary analysis of a phase II randomized trial Radiation Therapy Oncology Group (RTOG) 0212: impact of different total doses and 
schedules of prophylactic cranial irradiation on chronic neurotoxicity and quality of life for patients with limiteddisease small-cell lung cancer. Int J Radiat Oncol Biol Phys. 2011; 81(1): 77-84, doi: 10.1016/j.ijrobp.2010.05.013, indexed in Pubmed: 20800380.

4. Giuseppe ZR, Silvia C, Eleonora F, et al. Hippocampalsparing radiotherapy and neurocognitive impairment: A systematic literature review. J Cancer Res Ther. 2020; 16(6): 1215-1222, doi: 10.4103/jcrt.JCRT_573_17, indexed in Pubmed: 33342776.

5. Gondi V, Pugh SL, Tome WA, et al. Preservation of memory with conformal avoidance of the hippocampal neural stem-cell compartment during whole-brain radiotherapy for brain metastases (RTOG 0933): a phase II multi-institutional trial. J Clin Oncol. 2014; 32(34): 3810-3816, doi: 10.1200/JCO.2014.57.2909, indexed in Pubmed: 25349290.

6. Slotman B, Faivre-Finn C, Kramer G, et al. EORTC Radiation Oncology Group and Lung Cancer Group. Prophylactic cranial irradiation in extensive small-cell lung cancer. N Engl J Med. 2007; 357(7): 664-672, doi: 10.1056/NEJMoa071780, indexed in Pubmed: 17699816.

7. Takahashi T, Yamanaka T, Seto T, et al. Prophylactic cranial irradiation versus observation in patients with extensivedisease small-cell lung cancer: a multicentre, randomised, open-label, phase 3 trial. Lancet Oncol. 2017; 18(5): 663-671, doi: 10.1016/S1470-2045(17)30230-9, indexed in Pubmed: 28343976.

8. Rusthoven CG, Yamamoto M, Bernhardt D, et al. Evaluation of First-line Radiosurgery vs Whole-Brain Radiotherapy for Small Cell Lung Cancer Brain Metastases: The FIRE-SCLC Cohort Study. JAMA Oncol. 2020; 6(7): 1028-1037, doi: 10.1001/jamaoncol.2020.1271, indexed in Pubmed: 32496550.

9. Brown PD, Pugh S, Laack NN, et al. Radiation Therapy Oncology Group (RTOG). Memantine for the prevention of cognitive dysfunction in patients receiving whole-brain radiotherapy: a randomized, double-blind, placebo-controlled trial. Neuro Oncol. 2013; 15(10): 1429-1437, doi: 10.1093/neuonc/not114, indexed in Pubmed: 23956241.

10. Liu SV, Reck M, Mansfield AS, et al. IMpower133 Study Group. First-Line Atezolizumab plus Chemotherapy in Extensive-Stage Small-Cell Lung Cancer. N Engl J Med. 2018; 379(23): 2220-2229, doi: 10.1056/NEJMoa1809064, indexed in Pubmed: 30280641.
11. Gondi V, Tome WA, Rowley H, et al. Hippocampal contouring: A contouring atlas for RTOG 0933. 2010. http:// www.rtog.org..

12. The International Commission on Radiation Units and Measurements. J ICRU. 2010;10: Report 83. https://doi. org/10.1093/jicru/ndq001.

13. Gui C, Chintalapati N, Hales RK, et al. Prospective Study of Hippocampal-Sparing Prophylactic Cranial Irradiation in Limited-Stage Small Cell Lung Cancer. Int J Radiat Oncol Biol Phys. 2017; 98(3): 603-611, doi: 10.1016/j. ijrobp.2017.03.009, indexed in Pubmed: 28581401.

14. Belderbos J, Phd Md, Ruysscher DDe, et al. OC-0503 Phase III trial of Prophylactic Cranial Irradiation with or without Hippocampus Avoidance in SCLC. Radiother Oncol. 2019; 133: S259, doi: 10.1016/s0167-8140(19)30923-5.

15. Vees H, Caparrotti F, Eboulet El, et al. Swiss Group for Clinical Cancer Research (SAKK). Impact of Early Prophylactic Cranial Irradiation With Hippocampal Avoidance on Neurocognitive Function in Patients With Limited Disease Small Cell Lung Cancer. A Multicenter Phase 2 Trial (SAKK 15/12). Int J Radiat Oncol Biol Phys. 2020; 107(2): 279-287, doi: 10.1016/j.ijrobp.2020.02.029, indexed in Pubmed: 32142869.

16. Rodríguez de Dios N, Couñago F, López JL, et al. Treatment Design and Rationale for a Randomized Trial of Prophylactic Cranial Irradiation With or Without Hippocampal Avoidance for SCLC: PREMER Trial on Behalf of the Oncologic Group for the Study of Lung Cancer/Spanish Radiation Oncology Group-Radiation Oncology Clinical Research Group. Clin Lung Cancer. 2018; 19(5): e693-e697, doi: 10.1016/j. cllc.2018.05.003, indexed in Pubmed: 29891263.

17. ClinicalTrials.gov Identifier: NCT02906384. Memory and MRI Changes With Hippocampus Avoidance Prophylactic Cranial Irradiation(HA-PCI) for Small Cell Lung Cancer(SCLC). https://clinicaltrials.gov/ct2/show/ NCT02906384.

18. Gondi V, Pugh S, Mehta M, et al. NRG Oncology CC003: A randomized phase II/III trial of prophylactic cranial irradiation with or without hippocampal avoidance for small cell lung cancer. J Clin Oncol. 2019; 37(15_suppl): TPS8578-TPS8578, doi: 10.1200/jco.2019.37.15_suppl. tps8578.

19. Common Terminology Criteria for Adverse Events (CTCAE) Version 5.0 Published: November 27, 2017. https://ctep. cancer.gov/protocoldevelopment/electronic_applications/docs/CTCAE_v5_Quick_Reference_8.5x11.pdf. 